\title{
Descripción anatómica de las placas de Peyer en el intestino delgado de la alpaca (Vicugna pacos)
}

\author{
Anatomic description of Peyer's patches in the small intestine of the alpaca \\ (Vicugna pacos)
}

\author{
Jimmy Flores M. ${ }^{1,2}$, Miluska Navarrete Z. ${ }^{1}$, Alberto Sato S. ${ }^{1}$
}

\section{Resumen}

\begin{abstract}
Con el objetivo de describir las características anatómicas de las placas de Peyer en la alpaca, se recolectó el intestino delgado de 10 alpacas adultas aparentemente sanas, en el camal municipal de Huancavelica, Perú. El mayor número de placas de Peyer se observó en yeyuno, seguido de duodeno e íleon $(\mathrm{p}<0.05)$, pero la mayor densidad fue observada en el íleon $(\mathrm{p}<0.05)$. No hubo diferencias significativas por efecto del sexo de los animales. La ubicación con referencia al plano transversal del intestino fue de $65.88 \%$ en el borde anti-mesentérico, de $27.87 \%$ en el borde lateral y de $11.40 \%$ en el borde mesentérico $(\mathrm{p}<0.05)$. No se observó un patrón de distribución con referencia al plano longitudinal, excepto a nivel de la válvula ileal (2-3 placas de Peyer tipo quiste bordeando el anillo ileal). Se observaron varias formas de presentación: la forma nodular en duodeno y yeyuno, la faviforme en yeyuno, la de copa en yeyuno, íleon y esporádicamente en duodeno, y las más prominentes, la forma de quiste en las porciones distales del íleon. Además, se observaron dos formas intermedias: nodular-copa y copa-faviforme. Se concluye que la alpaca presenta características peculiares en las placas de Peyer diferentes a otras especies domésticas.
\end{abstract}

Palabras clave: alpaca, anatomía, placas de Peyer

\section{AbSTRACT}

The small intestine of 10 apparently healthy adult alpacas was collected in the municipal slaughterhouse of Huancavelica, Peru to describe the anatomical characteristics of Peyer's patches. The highest number of Peyer's patches was observed in the jejunum,

\footnotetext{
${ }^{1}$ Laboratorio de Anatomía Animal y Fauna Silvestre, Facultad de Medicina Veterinaria, Universidad Nacional Mayor de San Marcos, Lima, Perú

${ }^{2}$ E-mail: serolfjimmy@gmail.com
}

Recibido: 23 de octubre de 2019

Aceptado para publicación: 20 de junio de 2020

Publicado: 11 de agosto de 2020 
followed by the duodenum and ileum ( $<<0.05$ ), but the highest density was observed in the ileum $(p<0.05)$. There were no significant differences by effect of the sex of the animals. The location with reference to the transverse plane of the intestine was $65.88 \%$ on the anti-mesenteric border, $27.87 \%$ on the lateral border and $11.40 \%$ on the mesenteric border $(\mathrm{p}<0.05)$. No clear pattern of distribution with reference to the longitudinal plane was found, except at the level of the ileal valve (2-3 cyst shape Peyer's patches bordering the ileal ring.) Several forms of presentation were observed: the nodular form in the duodenum and jejunum, faviform form in jejunum, cup shape in jejunum, ileum and sporadically in duodenum, and cyst shape, the most prominent, in the distal portions of the ileum. In addition, two intermediate forms were observed: nodular-cup and cupfaviform. It is concluded that the alpaca presents peculiar characteristics in the Peyer's patches different from other domestic species.

Keys words: alpaca, anatomy, Peyer's patches

\section{INTRODUCCIÓN}

El intestino delgado representa una de las mayores superficies del organismo expuesta a patógenos, por lo que cuenta con una compleja morfología de defensa, en la que destacan el tejido linfoide asociado a intestino ( $G A L T$, por sus siglas en inglés) o placas de Peyer. Estas estructuras son parte importante de los mecanismos de inmunidad adquirida en el sistema digestivo, con características particulares para cada especie animal, encontrándose ubicadas a lo largo del yeyuno e íleon (Tizard, 2009).

Los primeros reportes de placas de Peyer en alpacas las describen como estructuras de diferentes formas y dimensiones, que van desde formas grandes y ovaladas $(2 \mathrm{~cm})$ hasta pequeñas e imperceptibles $(2 \mathrm{~mm})$. En adultos, se reportaron en duodeno, yeyuno y en el íleon a nivel de la porción ileocecal aquí, las cuales llegaron a ser lo suficientemente grandes como para obliterar las vellosidades del íleon y formar cráteres linfáticos (De la Vega, 1951; Montalvo, 1966; Fernández et al., 1999).

Actualmente se conoce que el intestino delgado de la alpaca es inmunológicamente muy activo; habiéndose descrito a las células de Paneth como elementos indispensables en la respuesta inmune innata (Lira et al., 2012; Vásquez et al., 2016). Las placas de Peyer han sido observadas al nacimiento en íleon y yeyuno y con dinámica linfoide diferenciada en las primeras semanas de vida (Roca et al., 2014); mientras que Uhde et al. (2017) llegaron a evaluar 14 anticuerpos marcadores de linfocitos y otras células especializadas en el GALT y otros órganos linfoides. Empero, no se ha llegado a estandarizar los criterios anatómicos de observación en cuanto a clasificación de forma, ubicación y medidas biométricas, de allí que en el presente trabajo se describen aspectos anatómicos de las placas de Peyer en el intestino delgado de esta especie.

\section{Materiales y Métodos}

Se seleccionaron mediante muestreo por conveniencia a 10 alpacas adultas mayores de 3 años, machos y hembras, de la raza Huacaya, sin evidencia clínica de enfermedad, durante los meses de enero y marzo de 2019. Los animales fueron muestreados en el camal municipal de Huancavelica, Perú. El cálculo de la edad adulta se determinó mediante cronología dentaria propuesto por Fowler (2010). Las vísceras del aparato di- 
gestivo se fijaron en formalina al 10\% y se remitieron al laboratorio de Anatomía animal y Fauna Silvestre de la Facultad de Medicina Veterinaria de la Universidad Nacional Mayor de San Marcos, en Lima.

La evaluación macroscópica consistió en observación ex situ, mediante disección y microdisección según Gil et al. (2012) y Pérez et al. (2016). Se localizó el intestino delgado, se delimitó y separó el duodeno, yeyuno e íleon, retirándose el mesenterio para registrar la biometría del intestino delgado. Se incidió el borde mesentérico para retirar el contenido intestinal mediante lavado con agua y exponer la luz intestinal. Se registró la forma de presentación de placas de Peyer de acuerdo con la clasificación propuesta por ShanShan et al. (2011). Asimismo, se registró la cantidad, ubicación y biometría con ayuda de calibre tipo Vernier. La densidad se calculó dividiendo la cantidad de placas de Peyer entre el área de observación. La descripción anatómica fue realizada de acuerdo con las pautas dadas por la Nomenclatura Anatómica Veterinaria (ICVGAN, 2005).

Para la confirmación de la presencia de agregados linfoides en las estructuras anatómicas se remitieron muestras para la evaluación histológica según los protocolos establecidos por Bancroft (Suvarna et al., 2012). Se tomaron fotografías con una cámara Canon HD35X y editadas con Adobe Photoshop 6.0. Los datos obtenidos fueron analizados con el programa IBM SSPS Statitics v. 22.0

El material biológico utilizado provino de animales de descarte de comunidades alpaqueras que llegaron al camal. El protocolo del trabajo fue aprobado por el Comité de Ética y Bienestar Animal (CEBA) de la Facultad de Medicina Veterinaria de la UNMSM (Constancia de Aprobación N. ${ }^{\circ}$ 2018-004)

\section{Resultados}

La observación anatómica de las placas de Peyer muestra que estuvieron distribuidas en los tres segmentos del intestino delgado de la alpaca, siendo la mayor cantidad promedio en el yeyuno, seguido de duodeno e íleon, con diferencia significativa entre segmentos $(p<0.05)$. Sin embargo, hubo una mayor densidad en íleon con respecto a yeyuno y duodeno $(\mathrm{p}<0.05)$. Por otro lado, no se observó diferencias significativas entre sexos.

En la distribución de las placas de Peyer en el intestino delgado, considerando el plano transversal del órgano, se observó una mayor presentación en el borde anti-mesentérico $(89.60 \pm 29.32)$, que representó el $65.88 \%$, seguido del borde lateral $(37.90 \pm 13.25) \mathrm{y}$ del borde mesentérico (15.50 \pm 7.07$)$, habiendo diferencias significativas $(\mathrm{p}<0.05)$ entre cada borde de intestino (Cuadro 2, Figura 1B).

Al considerar el plano longitudinal del intestino delgado, se observó que la distribución de las placas de Peyer fue aleatoria a lo largo de toda la extensión del órgano, sin seguir ningún patrón de ubicación referencial ni equidistante entre una u otra placa, con excepción de las ubicadas alrededor de la válvula ileal donde se observaron muchas veces paralelas o separadas a escasos milímetros de distancia hacia la parte craneal, todas ellas en forma de quiste (Figura 1A).

La distribución de las formas anatómicas de las placas de Peyer se presenta en el Cuadro 3. Se encontró diferencia significativa entre el número de cada forma $(\mathrm{p}<0.05)$, excepto entre Faviforme y Copa.

La forma nodular de placas de Peyer se caracterizó por ser estructuras a manera de proyecciones nodulares que sobresalieron 
Cuadro 1. Placas de Peyer y su distribución en el intestino delgado de 10 alpacas

\begin{tabular}{lccc}
\hline \multirow{2}{*}{ Alpacas } & \multicolumn{3}{c}{ Segmento de intestino delgado } \\
\cline { 2 - 4 } & $\begin{array}{c}\text { Duodeno } \\
(\text { media } \pm \text { DE) }\end{array}$ & $\begin{array}{c}\text { Yeyuno } \\
(\text { media } \pm \text { DE) }\end{array}$ & $\begin{array}{c}\text { Íleon } \\
\text { (media } \pm \text { DE) }\end{array}$ \\
\hline Machos & $91.0 \pm 17.9$ & $807.6 \pm 84.0$ & $21.1 \pm 2.6$ \\
Longitud $(\mathrm{cm})$ & $3.10 \pm 0.41$ & $3.24 \pm 0.52$ & $3.12 \pm 0.38$ \\
Diámetro $(\mathrm{cm})$ & $281.2 \pm 61.3$ & $2655.0 \pm 737.4$ & $65.1 \pm 4.1$ \\
Área $\left(\mathrm{cm}^{2}\right)$ & $23.6 \pm 8.4^{\mathrm{a} 1}$ & $158.2 \pm 51.7^{\mathrm{b} 2}$ & $12.8 \pm 5.4^{\mathrm{c} 3}$ \\
Placas de Peyer $(\mathrm{n})$ & $0.09 \pm 0.04^{\mathrm{a} 1}$ & $0.07 \pm 0.04^{\mathrm{a} 2}$ & $0.20 \pm 0.09^{\mathrm{b} 3}$ \\
Densidad $\left(\right.$ unidad/cm $\left.{ }^{2}\right)$ & & & \\
Hembras & $95.4 \pm 11.1$ & $782.6 \pm 75.5$ & $23.1 \pm 5.5$ \\
Longitud $(\mathrm{cm})$ & $2.96 \pm 0.23$ & $2.86 \pm 0.66$ & $2.65 \pm 0.12$ \\
Diámetro $(\mathrm{cm})$ & $283.4 \pm 46.9$ & $2268.7 \pm 672.6$ & $61.3 \pm 15.3$ \\
Área $\left(\mathrm{cm}^{2}\right)$ & $20.6 \pm 3.4^{\mathrm{a} 1}$ & $153.6 \pm 21.3^{\mathrm{b} 2}$ & $9.6 \pm 4.2^{\mathrm{c} 3}$ \\
Placas de Peyer $(\mathrm{n})$ & $0.07 \pm 0.02^{\mathrm{a} 1}$ & $0.07 \pm 0.02^{\mathrm{a} 2}$ & $0.16 \pm 0.1^{\mathrm{b} 3}$ \\
Densidad $\left(\mathrm{unidad} / \mathrm{cm}^{2}\right)$ & & & \\
\hline
\end{tabular}

$a, b, c$ Superíndices diferentes dentro de filas indican diferencia significativa $(p<0.05)$

$1,2,3$ Superíndices diferentes dentro de columnas indican diferencia significativa $(p<0.05)$

Cuadro 2. Distribución de las placas de Peyer en el intestino delgado de 10 alpacas (plano transversal)

\begin{tabular}{lccc}
\hline & \multicolumn{3}{c}{ Borde de intestino } \\
\cline { 2 - 4 } & Mesentérico & Lateral & Anti-mesentérico \\
\hline Cantidad (unidad) & $15.50 \pm 7.07^{\mathrm{a}}$ & $37.90 \pm 13.25^{\mathrm{b}}$ & $89.60 \pm 29.32^{\mathrm{c}}$ \\
Frecuencia (\%) & $11.40^{\mathrm{a}}$ & $27.87^{\mathrm{b}}$ & $65.88^{\mathrm{c}}$ \\
\hline
\end{tabular}

$a, b, c$ Superíndices diferentes dentro de filas indican diferencia significativa $(p<0.05)$

la mucosa intestinal, cubierta por una superficie rugosa que se asemejó a una uniforme y fina redecilla (Figura 2A). Fueron las más pequeñas en este estudio (Cuadro 3) y confirmadas por histología. Se ubicaron principalmente a partir del asa proximal del duodeno hasta las primeras porciones del yeyuno región proximal, camufladas de manera eficaz entre los pliegues y las vellosidades de la mucosa intestinal.

La forma faviforme se caracterizó por presentar proyecciones aplanadas que apenas sobresalieron el relieve de la mucosa del 


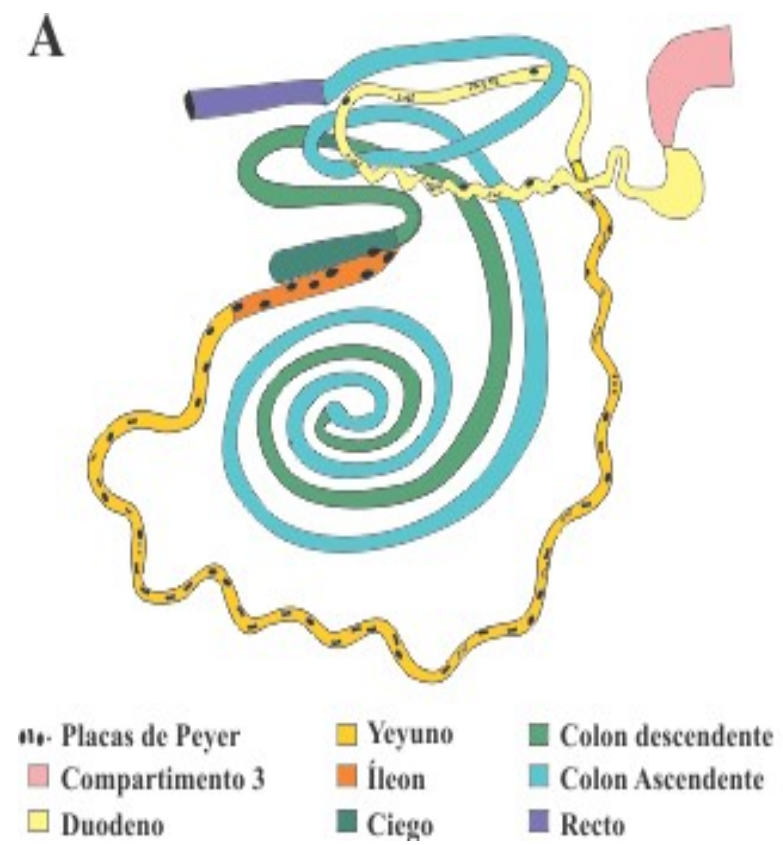

B
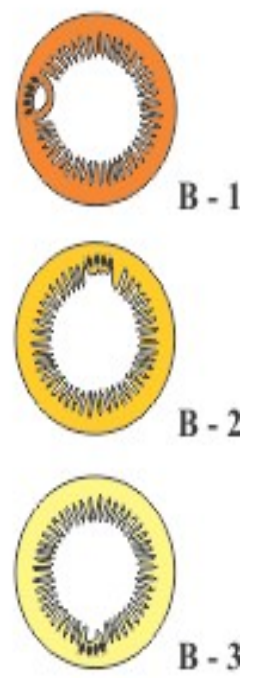

Figura 1. Distribución de las placas de Peyer en el intestino delgado de la alpaca. A Plano longitudinal. B Plano transversal. B-1: Forma de quiste en borde lateral; B-2: Faviforme en borde anti-mesentérico; B-3: Forma de copa en borde mesentérico (Fuente propia)

intestino. De superficie rugosa a manera de fina malla; detectables con mayor facilidad a trasluz. Se observó una gran variedad en las formas, habiendo formas circulares, alargadas, ovaladas, rectangulares o simplemente irregulares. De tamaño variable (Cuadro 3, Figura 2B). Se ubicaron exclusivamente en yeyuno, en especial a nivel de las zonas de mayor curvatura de las asas intestinales, lugar donde la mucosa intestinal careció de pliegues o vellosidades y las paredes fueron más delgadas.

La forma de copa se caracterizó por ser más perceptibles en forma y tamaño que las anteriores (Cuadro 3, Figura 2C). Su diseño estructural constó de un anillo ovalado que sobresale de manera notoria al relieve de la mucosa, que circunda una cavidad de fondo plano y tapizado por una superficie que se asemeja a una fina redecilla, similar a los ca- sos anteriores. Se le observó mayormente en yeyuno y en la región anterior del íleon, aunque en algunos animales se observó en duodeno. Por otro lado, se puede indicar que se ubicaron en áreas con abundantes vellosidades intestinales, situándose en medio de estas.

La forma de quiste fue la más prominente y llamativa, debido a su diseño anatómico particular que se asemejó a un sáculo ciego voluptuoso y alargado en dirección paralela al tubo intestinal adherida firmemente a la mucosa. Todas las placas de Peyer de esta forma presentaron una abertura u orificio de salida a manera de cráter, generalmente en el extremo distal del cuerpo (Figura 2D). El color fue similar a la mucosa intestinal, solo en un animal se observó coloración de tipo pardo rojiza. El tamaño promedio se presenta en el Cuadro 3. La ubicación fue exclusi- 


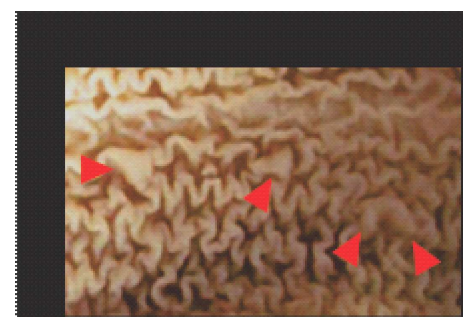

A

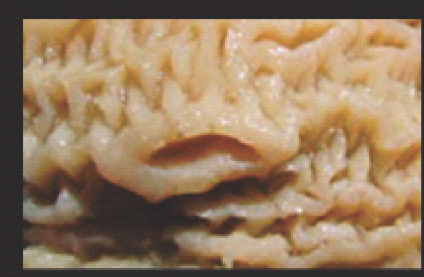

C - 1

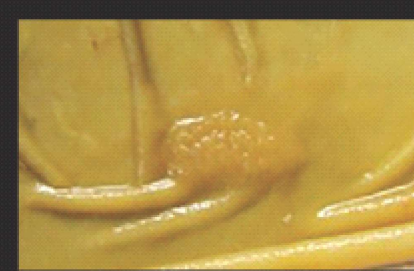

B - 1

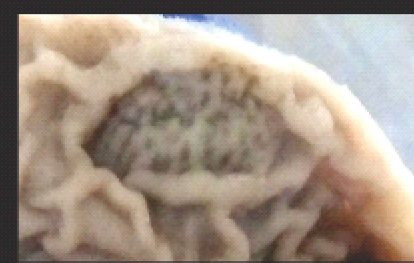

C - 2

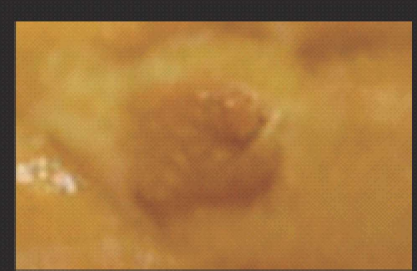

B - 2

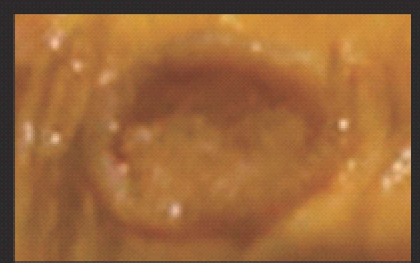

C - 3

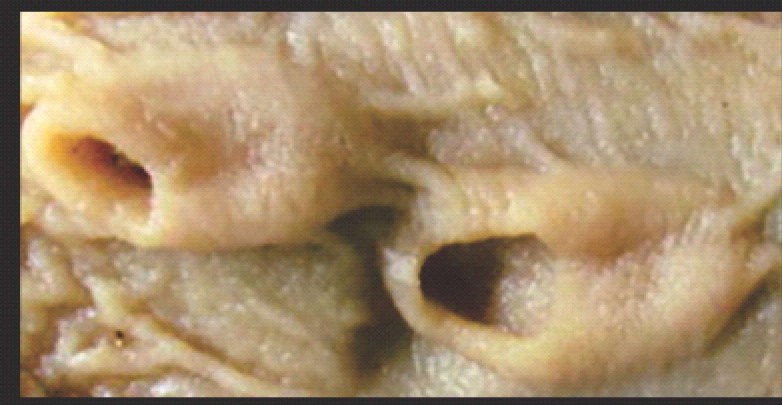

D - 1

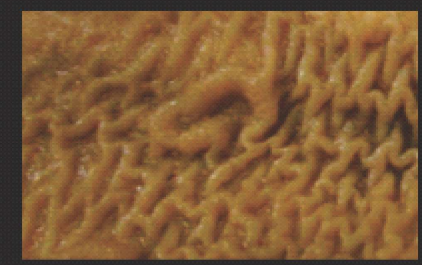

E - 1

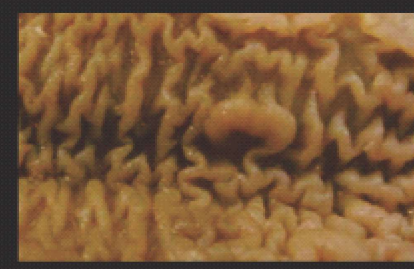

E - 2

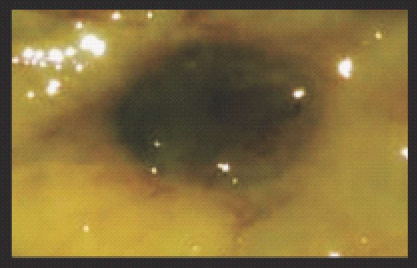

D - 2

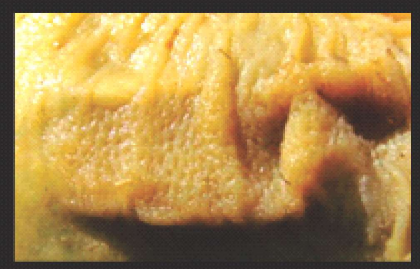

E - 3

$1 \mathrm{~cm}$.

Figura 2. Formas de placas de Peyer de la alpaca. $\mathrm{A}=$ Nodular en duodeno; B-1 y B-2 = Faviforme ovalada y cuadrangular en yeyuno; $\mathrm{C}-1=$ Copa en duodeno; $\mathrm{C}-2=\mathrm{Copa}$ en yeyuno; C-3 = Copa en íleon; D-1 = Quiste continuas en íleon; D-2 = Quiste con pigmentación; E-1 y E-2 = Intermedio entre nodular - copa; E-3 = Intermedio entre copa y faviforme 
Cuadro 3. Distribución de tipo y formas de las placas de Peyer en 10 alpacas

\begin{tabular}{|c|c|c|c|c|}
\hline \multirow[b]{2}{*}{ Forma } & \multirow{2}{*}{$\begin{array}{l}\text { Número } \\
\text { (n) }\end{array}$} & \multirow{2}{*}{$\begin{array}{c}\text { Frecuencia } \\
(\%)\end{array}$} & \multicolumn{2}{|c|}{ Medidas } \\
\hline & & & $\begin{array}{l}\text { Largo } \\
(\mathrm{mm})\end{array}$ & $\begin{array}{c}\text { Ancho } \\
\text { (mm) }\end{array}$ \\
\hline Nodular & $39.4 \pm 32.5^{1}$ & 20.9 & $3.9 \pm 1.4$ & $3.6 \pm 1.6$ \\
\hline Faviforme & $57.9 \pm 32.3^{2}$ & 30.6 & $12.4 \pm 7.6$ & $5.4 \pm 2.6$ \\
\hline Copa & $54.4 \pm 25.1^{2}$ & 28.8 & $10.4 \pm 4.3$ & $5.6 \pm 2.1$ \\
\hline Quiste & $4.7 \pm 1.3^{3}$ & 2.5 & $14.1 \pm 5.2$ & $7.1 \pm 2.4$ \\
\hline Intermedio & $19.7 \pm 10.8^{4}$ & 17.2 & $8.2 \pm 5.3$ & $4.6 \pm 2.1$ \\
\hline
\end{tabular}

$1,2,3,4$ Superíndices diferentes dentro de columnas indican diferencia significativa $(p<0.05)$

vamente en la porción distal del íleon distanciados en filas, de manera especial en todos los animales se observaron una a dos placas de Peyer adyacente inmediatamente al esfinter ileal.

En todos los animales se observaron dos formas intermedias de placas de Peyer. La primera entre las formas nodular y copa, cuya distribución fue principalmente en duodeno y parte anterior de yeyuno y la segunda variación fue entre copa y faviforme, distribuido principalmente en yeyuno (Figura 2E). Los tamaños fueron muy variables $(8.21 \pm 5.29$ de largo y $4.6 \pm 2.08$ de ancho).

\section{Discusión}

En las especies del Grupo I (rumiantes, cerdos, caballos, perros y hombre), entre el 80 y $90 \%$ de las placas de Peyer se encuentran en el íleon y se diferencian de las de yeyuno por ser más grandes y prominentes que involucionan a edad adulta. Por otro lado, en especies del grupo II (primates, conejos y roedores) son estructuras más reducidas que se distribuyen al azar, continúan su desarrollo hasta las 2-4 semanas después del nacimiento y persisten hasta la vejez (Liebler-Tenorio y Pabst, 2006; Pedrera et al., 2009; Tizard, 2009).
En camélidos del viejo mundo, la forma nodular, faviforme, copa y quiste se reportaron en Camelus bactrianus (Shan-Shan et al., 2011); mientras que, en Camelus dromedarius solo han sido reportadas las formas copa y faviforme (Alluwaimi et al., 1998; Zidan y Pabst, 2008). En este contexto, las placas de Peyer de la alpaca tienen mayor parecido a los animales del grupo II, mientras que en la comparación morfológica con las placas de Peyer de camélidos del viejo mundo presentan mayor similitud con las de Camelus bactrianus

En yeyuno e íleon de alpacas recién nacidas se han reportado placas de Peyer inmaduras con diferencias en su estructura histológica (Roca et al., 2014); por otro lado, Fernández, (1999) en animales adultos las describió como estructuras anatómicas de diferentes dimensiones y formas desde grandes y reconocibles hasta pequeñas e imperceptibles en algunos ejemplares. Así, en duodeno y yeyuno reportó tamaños desde $2 \mathrm{~mm}$ hasta de $2 \mathrm{~cm}$ y en íleon de $1.5-2 \mathrm{~cm}$. El presente estudio amplia la descripción y clasificación morfológica y corrobora con los resultados antes observados.

Estudios ontogénicos en animales indican que existen cambios estructurales en el desarrollo y maduración de los órganos del 
sistema inmune estrechamente relacionados con la edad (Tizard, 2009; Wang-Dong et al., 2012). En Camelus bactrianus, las placas de Peyer en animales jóvenes presentan insuficiente desarrollo, alcanzando el mayor grado de desarrollo a la edad adulta para luego decrecer e incluso desaparecer en animales gerontos (Shan-Shan et al., 2011). La observación de formas intermedias de placas de Peyer en este estudio, podría ser indicativo de que ciertos tipos de placas de Peyer son característicos para determinadas edades y que las placas de Peyer experimentan transición o cambian de forma conforme avanza la edad del animal.

\section{Conclusiones}

- La alpaca presenta placas de Peyer en las tres porciones de intestino delgado, y con mayor densidad en el íleon ( $\mathrm{p}<0.05)$, y sin diferencia por el sexo de los animales.

- La ubicación de las placas de Peyer con referencia al plano transversal del intestino fue de $65.88 \%$ en el borde antimesentérico, de $27.78 \%$ en el borde lateral y de $11.40 \%$ en el borde mesentérico $(\mathrm{p}<0.05)$.

- No se observó un patrón de distribución con referencia al plano longitudinal, excepto a nivel de la válvula ileal donde había placas de Peyer tipo quiste bordeando el anillo ileal.

- Las formas anatómicas de presentación de placas de Peyer son nodular en duodeno y yeyuno; faviforme en yeyuno; forma de copa en yeyuno, íleon y esporádicamente en duodeno y la forma de quiste, las más prominentes, únicamente en las porciones distales del íleon. Además, se observaron formas intermedias entre nodular y copa y entre copa y faviforme.

\section{Agradecimiento}

Los autores agradecen al FONDECYT CONCYTEC por el financiamiento del estudio mediante Convenio 025-2016.

\section{Literatura Citada}

1. Alluwaimi AM, Fath El-Bab MR, Ahmed AK, Ali AMA. 1998. Studies on the ileal lymphoid tissue (Peyer's patches) in Camels, Najdi sheep and cattle. J Camel Pract Res 5: 13-18.

2. De la Vega E. 1951. Aspectos histológicos del aparato digestivo y sistema urogenital de la alpaca. Tesis de Bachillerato. Lima, Perú: Univ. Nacional Mayor de san Marcos.

3. Fernández D, Tabacchi L, Sandoval N, Perales R, Santillán G. 1999. Caracterización de la estructura histológica de los órganos linfoides en alpacas (Lama pacos). Rev lnv Vet Perú 10: 110. doi: 10.15381/rivep.v10i2.6679

4. Fowler ME. 2010. Digestive system. In: Medicine and surgery of South American camelids. $2^{\text {nd }}$ ed. USA: Iowa State University Press. p 351-402.

5. Gil J, Gimeno M, Laborda J, Nuviala J. 2012. Protocolos de disección. $3^{\circ}$ ed. España: SERVET. $350 \mathrm{p}$.

6. [ICVGAN] International Committee on Veterinary Gross Anatomical Nomenclature. 2005. Nómina Anatómica Veterinaria (NAV). Hannover: ICGVAN.

7. Liebler-Tenorio EM, Pabst R. 2006. MALT structure and function in farm animals. Vet Res 37 257-280 doi: 10.1051/vetres:2006001

8. Lira MB, Cueva MS, Rodríguez, GJ, Ayón SM, Zanuzzi C, Barbeito C, Falcón PN, Vásquez CM. 2012. Identificación de las células de Paneth en el intestino delgado de alpacas en los primeros 21 días de edad. Rev Inv Vet Perú 23: 138-146. doi: 10.15381/rivep.v23i2.-893

9. Montalvo C. 1966. Contribución al estudio de la morfología de la alpaca (Lama pacos). Estudio histológico e histoquímico del intestino. Tesis de Médico Veterinario. Lima, Perú: Univ. Nacional Mayor de San Marcos. 55 p. 
10. Pedrera M, Sanchez PJ, Risalde MA, Molina V, Ruiz-Villamor E, RomeroPalomo F, Gomez-Villamandos F., 2009. El tejido linfoide asociado a mucosas como blanco del virus de la diarrea vírica bovina: Estructura y evolución. Anales Real Acad Cienc Vet Andalucía Oriental 22: 200-205.

11. Pérez W, König HE. Jerbi H, Clauss M. 2016. Macroanatomical aspects of the gastrointestinal tract of the alpaca (Vicugna pacos Linnaeus, 1758) and dromedary (Camelus dromedarius Linnaeus, 1758). Vertebrate Zool 66: 419-425.

12. Roca V, Manchego A, Sandoval N, Lam Chiok K, Rivera H. 2014. Caracterización histológica y dinámica linfoide de las placas de Peyer en crías de alpaca durante los 45 primeros días de vida. Rev Inv Vet Perú 25: 341-349. doi: 10.15381/rivep.v25i3.10112

13. Tizard I. 2009. Inmunología veterinaria. $8^{\mathrm{a}}$ ed. España: Elsevier. $591 \mathrm{p}$.

14. Shan-Shan Qi, Wen-Hui Wang, Qiang Gao, Xiao-Hong $\mathrm{Xu}$, Wan-Hong He, Ying-Pai Zhaxi, Li-Feng Tai. 2011. Age-related changes in the anatomical characteristics of Peyer's patches in small intestine of Bactrian camels (Camelus bactrianus). Trop Anim Health Prod 43: 1219-1223. doi: 10.1007/ s11250-011-9829-x
15. Suvarna KS, Layton C, Bancroft JD. 2012. Bancroft's theory and practice of histological techniques. $7^{\text {th }}$ ed. Elsevier Health Sciences. $654 \mathrm{p}$.

16. Uhde AK, Lehmbecker A, Baumgärtner W, Spitzbarth I. 2017. Evaluation of a panel of antibodies for the immunohistochemical identification of immune cells in paraffin-embedded lymphoid tissues of new- and old-world camelids. Vet Immunol Immunopathol. 184: 42-53. doi: 10.1016/j.vetimm.2017.01.001

17. Vásquez M, Lira B, Rodríguez J, Falcón N, Ocampo J, Nishida F, Barbeito C, Zanuzzi C. 2016. Characterization of Paneth cells in alpacas (Vicugna pacos, Mammalia. Camelidae). Tissue Cell 48: 383-388. doi: 10.1016/j.tice.2016.04.003

18. Wang-Dong Z, Wen-Hui W, XiaoHong $X$, Yin-Pai Z, Lin-Jiang Z, Shan-Shan Q, Hang L, Xue-Fen T. 2012. The histological characteristics of the aggregated lymphoid nodules area in abomasum of Bactrian camels (Camelus bactrianus) of different ages. Vet Immunol Immunopathol 147: 147-153. doi: 10.1016/j.vetimm.2012.04.007

19. Zidan M, Pasbt R. 2008. Unique microanatomy of ileal Peyer's patches of the one humped camel (Camelus dromedarius) is not age-dependent. Anat Rec 291: 1023-1028. doi: 10.1002/ar.20697 